\title{
Course of the Melting Transition in Organic Compounds
}

\author{
Francis J. Bartis \\ Department of Physics, Indiana University, Bloomington, IN 47405, USA \\ Z. Naturforsch. 46a, 630-634 (1991); received March 31, 1990
}

\begin{abstract}
This problem is reconsidered in the light of the mounting evidence that dislocations act as the centres of nucleation and growth. A new relation between the fraction of the sample in liquid form and the equilibrium temperature is derived and found to fit well the data on succinonitrile and $\mathrm{N}$-p-ethoxybenzylidene-p'-butylaniline (EBBA). It is argued that the additional dislocations in a cyclopentyl-1-thiaethane sample after rapid crystallization are responsible for the greater breadth of its melting transition compared to that of the same sample following slow crystallization. Past studies of the fractional melting process are criticized for their failure to include the early stages of the transition in their comparison of theory and experiment.
\end{abstract}

Key words: Melting, Latent heat, Organic compounds, Dislocations, Impurities.

\section{Introduction}

Our approach to the melting of organic compounds can be justified on several grounds. One is the limited success of the conventional approach. Assuming that impurities cause the latent heat to be consumed over a range of temperatures, investigators often obtain good fits over just the tail end of the melting process [1]. Another is the recently reported evidence of dislocation-initiated melting in several compounds $[2,3]$. A third reason is the claim by Mnyukh [4] that microcavities of some optimum size serve as the centres of nucleation in van der Waals bonded solids. Before throwing out all previous work, we should look into the possibility that melting may also start near dislocations. Our last reason is the strange position that dislocations occupy in current theories of crystallization and melting. Frank [5] maintains that dislocations play a pivotal role in crystal growth. His thesis finds support in observations on diverse materials [6-8]. Melting is just the reverse of crystallization. Yet dislocations are not even mentioned in the conventional approach to melting.

A simple model of second-phase growth underlies our approach. We take the spread in the melting temperature across a sample to be a consequence of the inhomogeneity of the stresses around crystal defects. For the sake of mathematical convenience, we treat the crystal lattice as an isotropic elastic continuum. The stress is then inversely proportional to the dis-

Reprint requests to Dr. F. J. Bartis, 15 Inwood Street, Dorchester, MA 02125, USA. tance from a dislocation [9] but inversely proportional to the cube of the distance from an impurity [10]. Assuming that the melting temperature varies linearly with the stress, we find that the radius of the cylindrical liquid domain alongside a dislocation goes as $\left(T_{\mathrm{M}}-T\right)^{-1}$, whereas the radius of the spherical liquid domain around an impurity goes as $\left(T_{\mathrm{M}}-T\right)^{-1 / 3}$. Here $T_{\mathrm{M}}$ denotes the nominal melting point.

\section{Theory}

Our model of second-phase growth enables us to make specific predictions for the singular features of the melting transition. An interesting case is presented by the nuclear magnetic resonance spectrum of thiophene near its nominal melting point. The investigators [11] observe a narrow proton line on top of the broad proton line corresponding to the crystalline phase. $\mathrm{A}\left(T_{\mathrm{M}}-T\right)^{-1.9}$ dependency is measured for the intensity of the narrow line. From the way the latent heat is consumed as a function of the temperature [12] it is evident that a minute amount of the liquid phase is present at temperatures below $T_{\mathrm{M}}$. Ascribing the narrow proton line to this minute quantity of liquid thiophene (cf. [13]), we expect the intensity of the narrow line to go as the volume of the liquid domains or as $\left(T_{\mathrm{M}}-T\right)^{-2}$ if the domains form along dislocations or as $\left(T_{\mathrm{M}}-T\right)^{-1}$ if the domains develop around impurities. We conclude that liquid thiophene makes its initial appearance near dislocations.

Our model of second-phase growth also enables us to give a formal description of the fractional melting 
process. We envisage a sample containing a fixed number of well separated defects. We need only realize that the fraction $F$ of the sample melted is proportional to the volume of the liquid domains. Recalling that the volume of the cylindrical domain alongside a dislocation goes as $\left(T_{\mathrm{M}}-T\right)^{-2}$, we can follow dislocationinitiated melting through

$$
T_{\mathrm{M}}-T=A F^{-1 / 2} .
$$

Likewise, remembering that the volume of the spherical domain around an impurity goes as $\left(T_{\mathrm{M}}-T\right)^{-1}$, we can trace impurity-initiated melting through

$$
T_{\mathrm{M}}-T=B F^{-1} .
$$

Physically we expect $A$ to depend on the dislocation content of the sample but $B$ to depend on its impurity content.

In our use of the foregoing equations we should be mindful of the various approximations made in their derivation. Conditions in the experimental sample should at least approximate those in our theoretical description of second-phase development. In our treatment of liquid domain growth each defect is supposed to be alone in an infinite crystal. To make sure that the sample behaves like an ensemble of solitary defects we must exercise care not only in the preparation of the sample but also in the selection of the material. It is not enough to note the excellent optical appearance of the sample when it is placed in the calorimeter. We should be wary of the possibility of severe plastic deformation during a prior polymorphic transformation or of thermal decomposition during the melting transition. In the latter instance the solitary-defect approximation is undermined by a varying impurity content, whereas in the former it is subverted by a high dislocation content.

We need not be concerned about our treatment of the crystal lattice as a continuous elastic medium. Admittedly the continuum approximation provides a poor description of the stresses in the immediate vicinity of the defects. So it is fortunate that these stresses influence a very miniscule portion of the transition. By way of illustration, let us consider a sample containing 2500 dislocations $/ \mathrm{cm}^{2}$. We estimate the maximum domain radius to be $10^{-2} \mathrm{~cm}$. In contrast we expect the stress field of a dislocation to deviate substantially from the prediction of the continuum theory below a distance of about $2 \times 10^{-7} \mathrm{~cm}$. It follows then that the continuum approximation introduces an error of
$4 \times 10^{-8} \%$ in our assessment of the course of the melting transition.

More worrisome is our assumption that the melting temperature varies linearly with the stress. Typical is the case of cyclohexane. From the calorimetric work of Aston et al. [14] we learn that $95 \%$ of the phase change occurs within 0.64 degrees Kelvin. Assuming that the plastic phase of cyclohexane is isotropic, we can obtain the stress dependence of the melting temperature directly from the measurements of its pressure dependence by Würflinger [15]. Thus we estimate that the stress dependence of the melting temperature varies some $0.1 \%$ over the main part of the phase change. We conclude that our supposition of a linear variation of the melting temperature with the stress introduces a small but acceptable error in our account of the fractional melting of cyclohexane.

We realize that through the above premise we risk a sizable error if the melting temperature as a function of the pressure passes through a maximum. But we see no signs of such a maximum in the published phase diagrams of organic compounds.

\section{Application}

We can make a good case for the application of (2) to the melting of highly refined metals. The underlying model of liquid domain growth around impurities accounts for the $\left(T_{\mathrm{M}}-T\right)^{-2}$ variation of the anomalous heat capacities of tin and indium [16-18]. Surprisingly, we can make a better case for the application of (1) to the melting of highly purified organic compounds. The underlying model of liquid domain growth alongside dislocations explains not only the $\left(T_{\mathrm{M}}-T\right)^{-2}$ dependence of the intensities of the narrow proton line in the NMR spectra of thiophene, cyclohexanol and benzene $[3,11]$ but also the $\left(P-P_{0}\right)^{-3}$ variation of the anomalous thermal expansivities of butane-1,4-diol and benzene $[2,19]$ and the $\left(T_{\mathrm{M}}-T\right)^{-2}$ mode in the self-diffusion of pivalic acid [3, 20]. To decide whether (1) or (2) is appropriate we appeal to both $F^{-1 / 2}$ vs. $T$ and $F^{-1}$ vs. $T$ plots. Ideally the data points should fall along a curve in one plot but along a straight line in the other. To test our new equation of fractional melting we use published data $[21,22]$ on succinonitrile and $\mathrm{N}$-p-ethoxybenzylidene-p'-butylaniline (EBBA). We find that their $F^{-1}$ vs. $T$ plots (or melting curves) more or less depart from straight lines. On the other hand, we can see in Figs. 1 and 2 that 


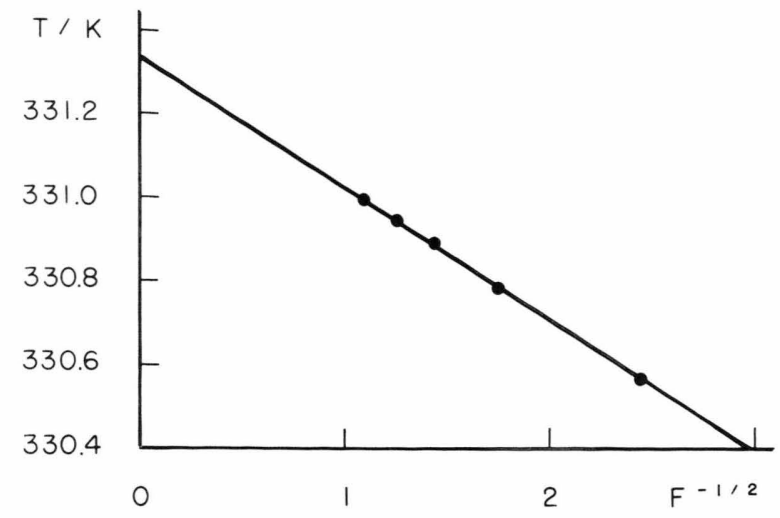

Fig. 1. Modified melting curve of succinonitrile.

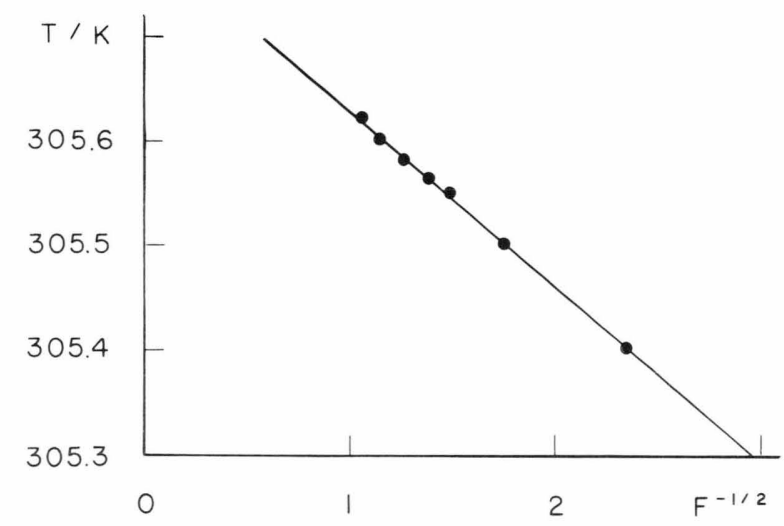

Fig. 2. Modified melting curve of EBBA.

their $F^{-1 / 2}$ vs. $T$ plots (or modified melting curves) yield straight lines. Thus we establish that (1) describes the fractional melting of both compounds and hence that dislocations are the sites of the liquid domains.

In light of the growing evidence for dislocationinitiated melting we must consider anew the effects of the thermal history of the sample on its melting curve. For example, a specimen of cyclopentyl-1-thiaethane rapidly crystallized is observed to undergo melting over a larger temperature interval than the same specimen slowly crystallized [1]. In the conventional approach to melting this dependence on the crystallization mode is accounted for by the presence of solidsoluble impurities in the specimen. It is argued that the crystallization rate determines the extent to which the impurities are incorporated into the lattice. We find more plausible an explanation in terms of the dislocation structure of the specimen. Numerous ex- perimental studies can be cited in support of a greater dislocation density following rapid crystallization. It is easy to see how the additional dislocations would lead to a large variation in the stresses acting on the various parts of the specimen and hence a wider spread in their melting temperatures.

To quantify the effect of the crystallization mode on the dislocation density it is sufficient to assume that at atmospheric pressure the size of the liquid domains in a given organic compound is determined solely by the ambient temperature. Accordingly, we take the domains at $T_{\mathrm{C}}=169.8498 \mathrm{~K}$ in a cyclopentyl-1-thiaethane sample after rapid crystallization to be identical in size to those at $T_{\mathrm{C}}$ following its slow crystallization. Experimentally it is found [1] that at $T_{\mathrm{C}}$ the liquid domains constitute $44.38 \%$ of the sample after fast crystallization but $14.34 \%$ of the sample after slow crystallization. Hence the number of liquid domains after rapid crystallization is 3.09 times the number after slow crystallization. Since a dislocation is associated with each domain, the dislocation density after fast crystallization must be 3.09 times the dislocation density following slow crystallization.

\section{Discussion}

Our findings with regard to the onset of melting in succinonitrile and EBBA are completely at odds with those of the original investigators [21, 22]. They claimed that the $F^{-1}$ vs. $T$ plot of succinonitrile was linear, whereas that of EBBA was curved. Taking the conventional approach to melting, they concluded that solid-insoluble impurities initiated the melting of succinonitrile, while solid-soluble impurities nucleated the transition in EBBA.

At first we are surprised by the disagreement regarding the onset of melting in succinonitrile. We wonder how a difference of opinion can arise. If (2) characterizes the melting of succinonitrile under the conventional scheme, then logically it should also do so under ours. Later we realize that we are utilizing more data than the original investigators. We suspect that they compared theory and experiment just during the later stages of the melting process. It is evident from Table 1 that the data are well approximated by a straight line in a $F^{-1}$ vs. $T$ plot covering the final two-tenths of a degree Kelvin but deviate from a straight line in such a plot extending over the last half of a degree Kelvin. 


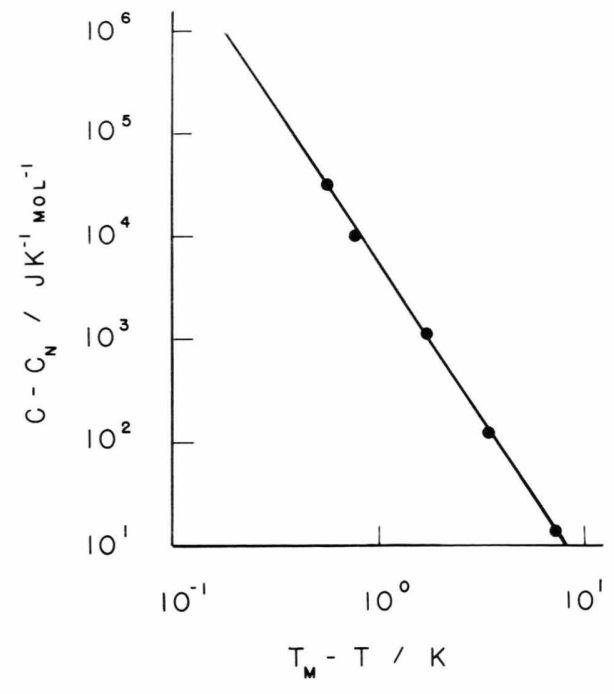

Fig. 3. Anomalous heat capacity of EBBA as a function of the relative temperature.

Table 1. Fractional melting of succinonitrile. $T_{\text {calc }}$ is obtained on the assumption that the temperature varies linearly with the inverse of the fraction melted.

\begin{tabular}{lcc}
\hline$F^{-1}$ & $T_{\text {calc }}$ & $T_{\text {obs }}$ \\
\hline 5.908 & $330.468 \mathrm{~K}$ & $330.566 \mathrm{~K}$ \\
3.068 & 0.781 & 0.781 \\
2.053 & 0.893 & 0.886 \\
1.551 & 0.948 & 0.948 \\
1.169 & 0.990 & 0.993 \\
\hline
\end{tabular}

The earlier investigation of EBBA is also open to criticism for its failure to take the early stages of the transition into account. Table 2 of [22] shows that the Mastrangelo-Dornte equation [23] describes well the course of the phase change during the final two-tenths of a degree Kelvin. But the precision of adiabatic calorimetry enables us to record the evolution of the latent heat of melting over almost ten degrees Kelvin. We take full advantage of the experimental information at hand, when we examine the divergent behaviour of the molar heat capacity of EBBA. Using our model of liquid domain growth, we can prove that the contribution to the molar heat capacity from the latent heat goes as $\left(T_{\mathrm{M}}-T\right)^{-3}$ when dislocations ini- tiate the transition but as $\left(T_{\mathrm{M}}-T\right)^{-2}$ when impurities nucleate the transition. The nominal melting point $T_{\mathrm{M}}$ is chosen so that the data points in a log-log plot of the anomalous heat capacity $\left(C-C_{\mathrm{N}}\right)$ versus the relative temperature $\left(T_{\mathrm{M}}-T\right)$ fall along a straight line. We estimate the normal heat capacity $C_{\mathrm{N}}$ by extrapolation of the data recorded well below the transition. With $T_{\mathrm{M}}=305.995 \mathrm{~K}$ we obtain the plot displayed in Figure 3 . The -3.02 slope of the straight line through the data points serves to identify dislocations as the initiators of the transition.

\section{Outlook}

We see a couple of areas worth exploring in future studies. We regard as an open question whether the centres of nucleation in the melting of highly refined samples are determined by the nature of the binding forces of the solids. It now appears that dislocations initiate the transition in molecular crystals, whereas impurities nucleate it in metals. But crystal growth studies point to a general competition between dislocations and impurities. For example, the growth velocity is found to be a double-valued function of the undercooling of the interphase boundary in gallium. It is thought that dislocation-assisted growth gives one value, while dislocation-free growth gives the other [24]. To make the case for a general competition, investigators should vary the experimental conditions and look for clear signs that dislocations are initiating the melting of gallium.

We should also give some thought to the bubble problem raised by Cummins and his colleagues [25]. They contend that tiny bubbles are responsible for the strong scattering of light by an advancing crystalliquid interface. They attribute the bubbles to air occluded in the crystal but released when it undergoes a rapid phase change. We see the same problem complicating the investigation of polymorphic transitions by optical microscopy. It may well have compromised the efforts of Mnyukh [4]. The microcavities that he took to be the centres of nucleation may just be tiny air bubbles formed at the moving interphase boundary. 
[1] J. P. McCullough and G. Waddington, Anal. Chim. Acta 17, 80 (1957).

[2] F. J. Bartis, Solid State Commun. 58, 653 (1986).

[3] F. J. Bartis, Phil. Mag. Lett. 60, 17 (1989).

[4] Yu. V. Mnyukh, J. Cryst. Growth 32, 371 (1976).

[5] F. C. Frank, Disc. Faraday Soc. 5, 48 (1949).

[6] H. Bethge, Phys. Stat. Sol. 2, 3 (1962).

[7] A. J. Forty, Phil. Mag. 43, 481 (1952).

[8] I. M. Dawson and V. Vand, Nature London 167, 476 (1951).

[9] H. G. van Bueren, Imperfections in Crystals, NorthHolland, Amsterdam 1960, p. 55.

[10] Ibid., p. 94.

[11] B. Rousseau, C. Bessada, and A. H. Fuchs, Solid State Commun. 67, 1017 (1988).

[12] P. Figuiere, H. Szwarc, M. Oguni, and H. Suga, J. Chem. Thermodyn. 17, 949 (1985).

[13] H. Suga, M. Sugisaki, and S. Seki, Mol. Cryst. 1, 377 (1966).

[14] J. G. Aston, G. J. Szasz, and H. L. Fink, J. Amer. Chem. Soc. 65, 1135 (1943).
[15] A. Würflinger, Ber. Bunsenges. Phys. Chem. 79, 1195 (1975).

[16] F. J. Bartis, Nature London 268, 427 (1977).

[17] F. Gronvold, Rev. Chim. Miner. 11, 568 (1974).

[18] F. Gronvold, J. Therm. Anal. 13, 419 (1978).

[19] F. J. Bartis, Phil. Mag. A 52, L 33 (1985).

[20] R. Freer, P. W. Salthouse, and J. N. Sherwood, Phil. Mag. A 45, 205 (1982).

[21] C. A. Wulff and E. F. Westrum Jr., J. Phys. Chem. 67, $2376(1963)$

[22] M. Sorai, T. Nakamura, and S. Seki, Bull. Chem. Soc. Japan 47, 2192 (1974).

[23] S. V. R. Mastrangelo and R. W. Dornte, J. Amer. Chem. Soc. 77, 6200 (1955).

[24] G. J. Abbaschian and S. F. Ravitz, J. Cryst. Growth 28, 16 (1975).

[25] H. Z. Cummins, G. Livescu, H. Chou, and M. R. Srinivasan, Solid State Commun. 60, 857 (1986). 